Communications in Physics, Vol.21, No. 3 (2011), pp. 235-243

\title{
DENSITY FUNCTIONAL BASED TIGHT BINDING STUDY ON WURTZITE ZNO PRISMATIC NANOPARTICLES
}

\author{
NGUYEN VIET MINH \\ Institute of Engineering Physics, \\ Hanoi University of Science and Technology (HUST) \\ VU NGOC TUOC AND LE THI HONG LIEN \\ Institute of Engineering Physics, HUST \\ and \\ International Center for Computational Materials Science, HUST
}

\begin{abstract}
We have performed the Density Functional Tight Binding (DFTB) study on the structural properties of Zinc Oxide Nanoparticles (NP), focusing on the effects induced by the surfaces and quantum size effect. Effects of surface relaxation and surface stress which is absent in atomistic model are taken carefully into account. The studying Nanoparticle size range up to $2.3 \mathrm{~nm}$. We illustrated the structural properties changes by decreasing NP sizes while the typical length of surface relaxation (about $1 \mathrm{~nm}$ ) remain unchanged and comparable with the particle size. The NP electronic properties, i.e. Density of State (DOS), charge transfer in hetero-bond also showed the deviation from bulk material value.
\end{abstract}

\section{INTRODUCTION}

Nanostructured $\mathrm{ZnO}$ materials have been the subject of intensive study owing to their distinguished performance in optoelectronics, sensors, transducers, and biomedical sciences. ZnO material has three key properties: (i) direct-wide bandgap $(3.37 \mathrm{eV} \tilde{3} 75 \mathrm{~nm}$ at $300 \mathrm{~K})$, polar surface, high exc. bind. energy $(60 \mathrm{meV})$ which may result in high efficient light emitting nano device (blue LED, nanoscale UV laser), photo-detector. Its transparent conductivity may find application in flat panel display, solar cell. (ii) the lack of a centre of symmetry in wurtzite, combined with its large electromechanical coupling, results in strong piezoelectric and pyroelectric properties and the consequent use of $\mathrm{ZnO}$ in surface acoustic wave device for communication and sensing applications; (iii) Its bio-safe and biocompatibility may result in many biomedical applications without coating. When doped with transition metals it can form spin-polarized light sources and could be diluted magnetic semiconductor.

Although it is known that properties of macrosize semiconductors are independent of their size, nevertheless recent studies on semiconductor nanoparticles (NP) [1-2] have shown with characteristic size of semiconductor particles is less or about $10 \mathrm{~nm}$, then their major physicochemical properties are essentially different from their of macroscopic objects and appear to be strongly size and shape dependent. This is due to the fact that semiconductors generally are characterized by high energies of intermolecular interactions 
and their electronic excitation results in the formation of a loosely coupled electron-hole (e-h) pair, with the size of the delocalization area of which, that characterized by the size of excitons Bohr radius, can be much larger than the lattice constant. Therefore decreasing size of them to a comparable size with e-h delocalization area will affects their electronic characteristics, e.g. blue shift of the excitonic absorption band of CdS from a crystal size about 5nm [2-3]. Further in a NP the surface-to-volume ratio of the atom involving species, e.g. for 5 -nm CdS NP it is about $15 \%$ compare to $0 \%$ in infinite bulk materials therefore quantum size effects are expected to have a dominant influence, e.g. significant detraction of the exciton binding energy.

Recently electronic quantum size effect in semiconductor quantum dots has been largely studied, and important optical device applications have been developed owing to their discrete atomiclike density of states (DOS). More recently, IIVI compounds as (e.g. ZnO) have attracted a strong interest for the realization of UV optoelectronic devices operating at $300 \mathrm{~K}$, since their high band gap and large exciton binding energies. In this work, we have performed the Density Functional Tight Binding (DFTB) study on the structural properties of Zinc Oxide ( $\mathrm{ZnO}) \mathrm{NP}$, focusing on the effects induced by the surfaces and quantum size effects. Structures of studies here are freestanding wurtzite $\mathrm{ZnO}$ prisms NP identical to the ones experimentally synthesized by a wet chemistry method (see [2-4] and reference there in). The choice of prismatic hexagonal shape also corresponds to the symmetry of the hexagonal lattice. To describe the effects of the surface, we compare structural properties of two series of ZnO NPs with the same cross section diameters (16.7 and $23.2 \AA$ ) and different logitudal size ranging from $11 \AA$ to $22 \AA$, i.e., approaching the experimental sizes.

Zinc-containing systems have been widely investigated by first-principles methods $[5,14,17-18]$, however it become difficult to treat a large number of atoms because of their high computational demands. Therefore, self-consistent charge density-functional tight-binding (SCC-DFTB) method [6-8], which has been successfully applied to largescale quantum-mechanical simulations, is suitable candidate for the task. The method is an approximation to the Kohn-Sham density-functional theory (DFT), which combines reasonable accuracy and computational efficiency. The present work represents an extensive study of $\mathrm{ZnO} / \mathrm{ZnS}$ hetero wurtzite systems, which we perform using first-principles Density Functional Tight-Binding calculations.

\section{DENSITY-FUNCTIONAL-BASED TIGHT-BINDING PLUS (DFTB+) METHOD}

The spin-polarized, charge self-consistent, DFTB approach is based on a secondorder expansion of the spin-dependent Kohn-Sham total energy functional with respect to a given reference charge and magnetization density. The method has been extensively discussed elsewhere [6-9] and briefly outlined here as:

1. Expand the orbitals as a linear combination of Slater type orbitals (LCSTO):

$$
\psi_{n}(\mathbf{r})=\sum_{\text {atomicsite } i} \sum_{\text {orbital } \nu} C_{i \nu} \phi_{i \nu}\left(\mathbf{r}-\mathbf{R}_{i}\right)
$$


The basis functions $\phi_{i \alpha}\left(\mathbf{r}-\mathbf{R}_{i}\right)$ centered on the atomic nucleus $i$, with position $\mathbf{R}_{i}$, are themselves a linear combination of single Slater orbitals

$$
\phi_{\nu}(\mathbf{r})=\left(\sum_{j=1}\left(\sum_{n=0} a_{j n} \mathbf{r}^{l_{\nu}+n}\right) e^{-\alpha_{j} r}\right) Y_{l_{\nu} m_{\nu}} .
$$

The angular and magnetic quantum number are indicated with $l_{\nu}$ and $m_{\nu}$. $Y_{l_{\nu} m_{\nu}}$ is the corresponding real spherical harmonic.

2. Tight-binding expansion of the wave functions (calculation of the matrix elements in the two-centers approximation).

$$
\sum_{\text {atomic site } i \text { orbital } \nu}\left|H_{i \nu, j \mu}-E S_{i \nu, j \mu}\right| C_{i \nu, j \mu}=0
$$

with

$$
H_{i \nu, j \mu}=\left\langle\phi_{i \nu}|\hat{H}| \phi_{j \mu}\right\rangle
$$

and

$$
S_{i \nu, j \mu}=\left\langle\phi_{i \nu} \mid \phi_{j \mu}\right\rangle
$$

3. Second order-expansion of Kohn-Sham energy functional (self-consistency in the charge density - SCC-DFTB):

$$
E_{\text {tot }}=\sum_{i}^{o c c .}\left\langle\psi_{i}\left|H_{0}\right| \psi_{i}\right\rangle+\frac{1}{2} \sum_{\mu, \nu} \gamma_{\mu \nu} \Delta q_{\mu} \Delta q_{\nu}+E^{r e p}
$$

where $\Delta q_{\mu}$ - charge fluctuation decomposed into atomic contribution (Mulliken charge), $\gamma_{\mu \nu}$ - some integral coefficient, $E^{r e p}$ - repulsive term (see [6-8]).

With all matrix elements and orbitals are derived from Density Functional calculation, the advantage of DFTB method relies on the use of small basic set of atomic orbitals (in order to reduce the matrix dimension for diagonalization speed-up) and the restriction to two center nonorthogonal Hamiltonian (allowing extensive use of look-up table). What it distinguishes from semi-empirical method is the explicite calculation of the basic wave function which allow deeper physics insight and better control of the approximation used. The method solved Kohn-Sham equation self-consistently using Mulliken charge projection. This approach have proved to give transferable and accurate interaction potential as well as numerical efficiency allowing Molecular Dynamic (MD) simulation of supercell containing several hundreds to a thousand atoms. Thus this is particular suitable to study the electronics proper and dynamics of large mesoscopic system and organic molecule such as CNTs, DNA stands or absorbate on surface, semiconductor hetero-structure etc. see review in [4-6]. The advantage of DFTB parameterization is that only few, possibly well chosen systems are needed to create the parameters, i.e. in DFTB fit systems can also be purely ideal systems, if they are chemically acceptable and can be described carefully with an ab-initio approach. Next this well tested parameter, e.g. in an attention for solid state systems and for defect physics as in current case, can be used for much larger system due to its transferability. In our calculation the parameter and its transferability have been successfully applied in several DFTB works $[10-12,16]$

The electronic DFTB parameters (i.e., Hubbard parameters, $\nu$ and $S_{\mu \nu}$ matrix elements) 
were derived directly from DFT calculations, performed within the generalized gradient approximation (GGA) and using the Perdew, Burke, and Ernzerhof (PBE) exchangecorrelation functional.

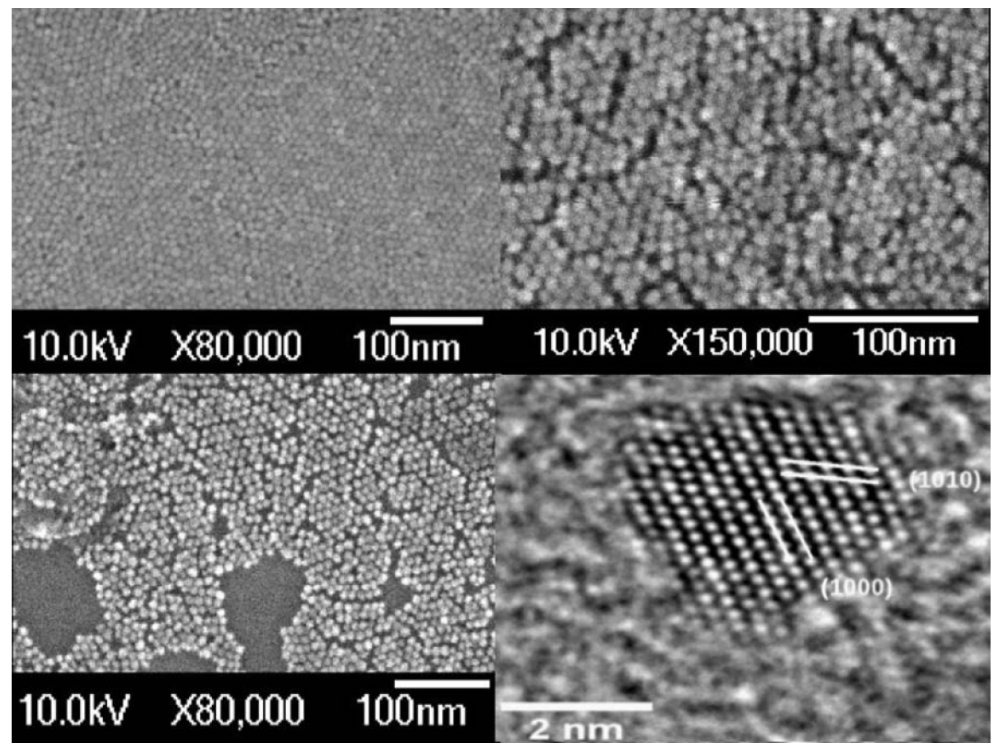

Fig. 1. SEM images of ZnO NPs synthesized following the route described in [3]. On the bottom right, HRTEM image of a NP in the [0001] direction.

\section{RESULTS AND DISCUSSION}

\section{III.1. Atomic structure}

In this work, we aim to simulate nanostructures of $\mathrm{ZnO}$ with a prismatic shape as synthesized experimentally (see Fig. 1). The prism axis is parallel to the c axis of the wurtzite lattice. We design a prismatic NP with hexagonal facets of wurzite crystal structure. Two series of $\mathrm{ZnO}$ hexagonal prismatic NP are created for simulation purpose with diameter $16.7 \AA$ (series A) and with diameter $23.2 \AA$ (series B) of three different prismatic hexagonal lengths each.

Fig. 2 shows the cross prismatic section perpendicular (left) and along prismatic, i.e. in [0001] direction, views of NPs fully optimized structures with description in Table 1.

For description of the NPs we use supercell wrapped with at least $10 \AA$ vacuum thickness to ensure there is no interaction of NP with their periodical image. In our pseudo potential approach, the Zn-3d electron states are treated explicitly as part of the valence band in order to take into account the hybridization between Zn-3d and O-2s. Therefore each Zinc atom site with have $6(5 d$ and $1 s)$ valence states with spin degeneration and Oxygen atom site with 3 valence states so that for example with the A-1 sample NPs with 216 atoms there are $(6+3) \times 108=972$ valence states (see the number of HOMO level in Table 1). 
Table 1. Stoichiometry of ZnO NPs

\begin{tabular}{cccc}
\hline \hline Series A & 1 & 2 & 3 \\
\hline Relaxed prismatic hexagonal length $(\AA)$ & 11 & 16.3 & 22 \\
\hline Total number of atom in NPs & 216 & 324 & 432 \\
\hline Highest Occupied Molecular Orbital $(\mathrm{HOMO})$ level & 972 & 1458 & 1944 \\
\hline Total number of Atomic Orbitals $($ AO) used & 1404 & 2106 & 2808 \\
\hline \hline Series B & 1 & & \\
\hline \hline Relaxed prismatic hexagonal length $($ A $)$ & 11 & 16.3 & 22 \\
\hline Total number of atom in NPs & 384 & 576 & 768 \\
\hline Highest Occupied Molecular Orbital $(\mathrm{HOMO})$ level & 1728 & 2592 & 3456 \\
\hline Total number of Atomic Orbitals $($ AO $)$ used & 2496 & 3744 & 4992 \\
\hline \hline
\end{tabular}
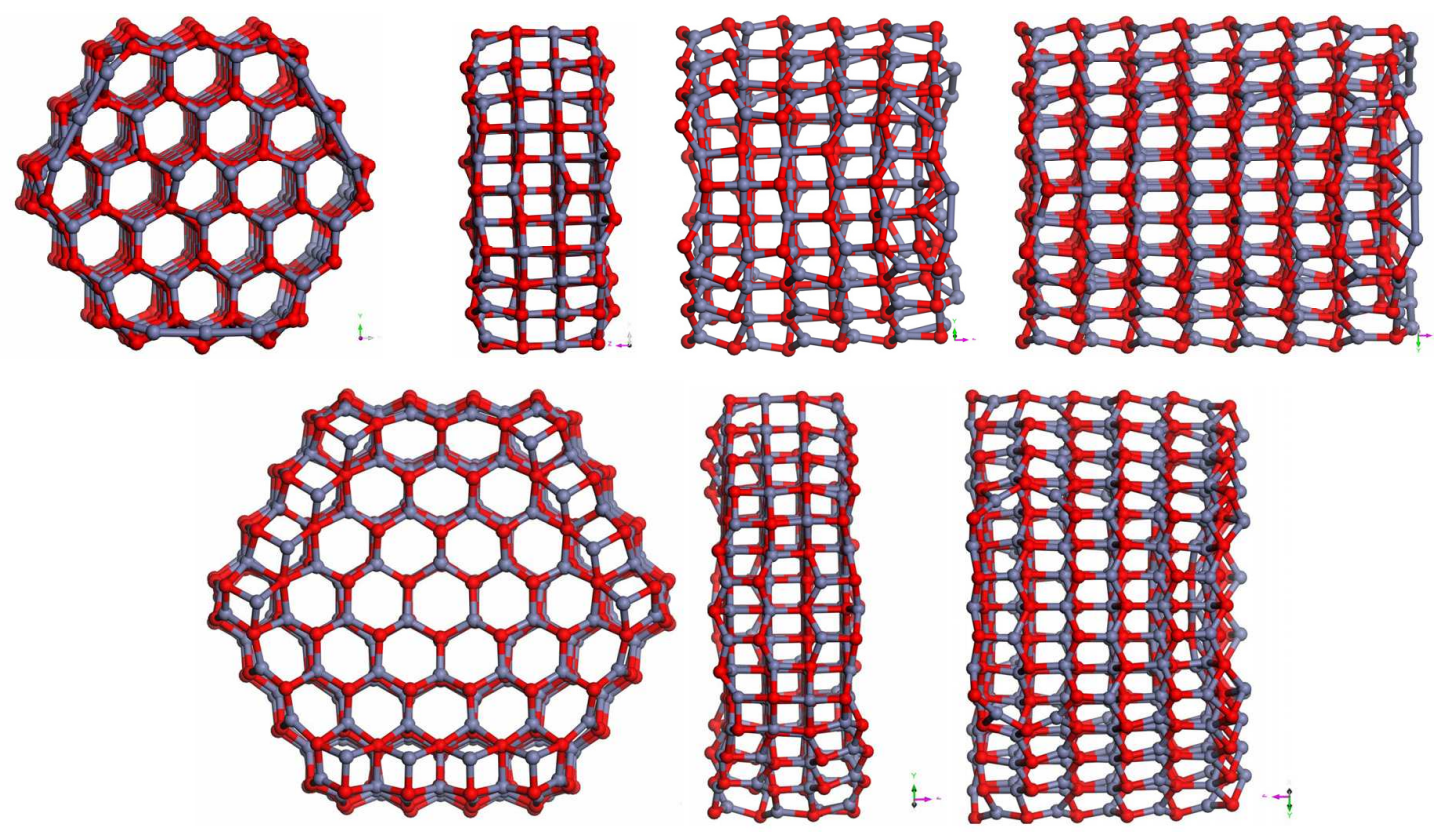

Fig. 2. (Color online) Core-shell $\mathrm{ZnO} / \mathrm{ZnS}$ heterostructure $\mathrm{NW}$ Nanowires in [0001] direction with fully optimized structures in cross section view.

By using localized basic sets, i.e. linear combination of atomic orbital LCAO, each O-site will have 4 (one s-orbital and three p-orbital) orbital and Zn-site will have 9 (one $s$-orbital, three $p$-orbital and five $d$-orbital) so that for example with the A-1 sample NPs with 216 atoms there are of $(4+9) \times 108=1404$ localizes atomic orbitals (see the number of Atomic Orbitals (AO) used in Table 1).

Top and bottom surfaces of the prism, which exhibit a nonzero dipole moment perpendicular to the surface due to the alternation of $\mathrm{O}$ and $\mathrm{Zn}$ layers creates a dipole that 
monotonically increases as a function of the crystal thickness and gives rise to a surface instability. Figures 2, 3, and 4 show that both series A and B show this trend from 1 to 3 by increasing the NPs thickness. However the Zn-facet, i.e. grey color atom facet or right facet in Fig. 2 is deformed under larger strain. In contrary the O-facet, i.e. left facet stabilized to more plain facet. This is due to the charge transfer between $\mathrm{Zn}$ and $\mathrm{O}$ at the facets. (see Fig. 2).

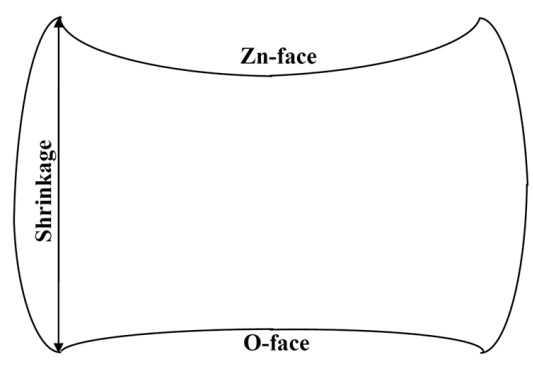

Fig. 3. Sketch of the relaxed NP shape of studied ZnO hexagonal prismatic NP.

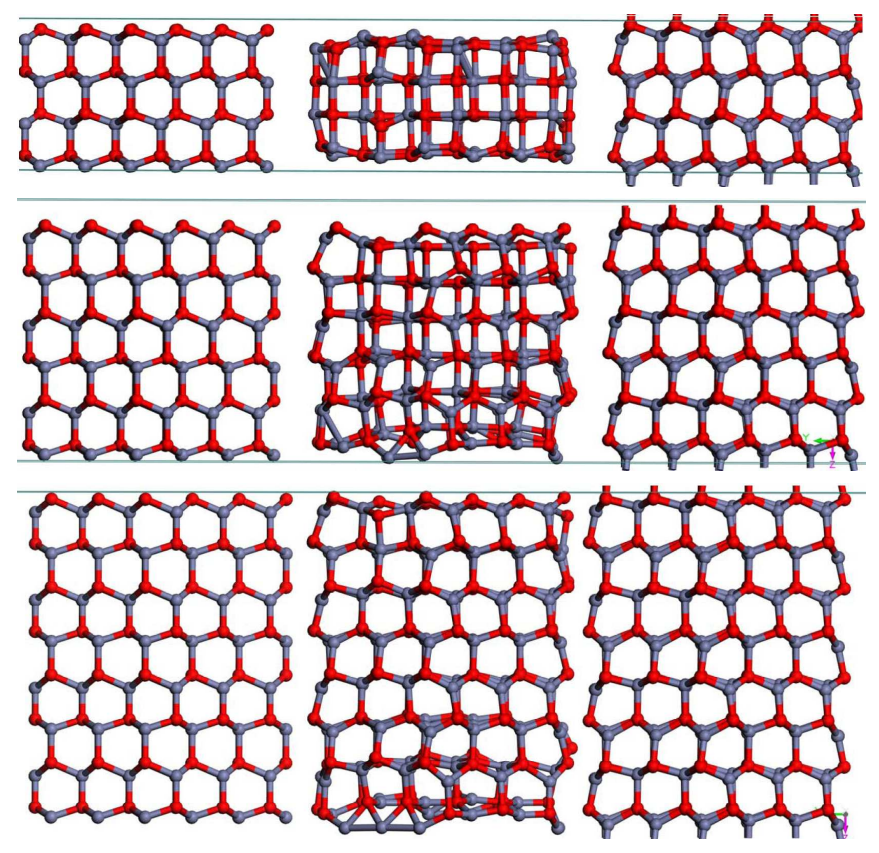

Fig. 4. (Color online) Comparable cross section view for $\mathrm{ZnO}$ bulk (left column), NPs (middle) and Nanowire [19] Purple axis denotes the $\mathrm{z}$ axis parallel to the $\mathrm{c}$ axis of the hcp crystal structure.

Fig. 3 shows the sketch of the NPs relaxation including the shrinking of cylindrical shape along radial direction due to the dangling bonds on the outer co-axial layer similar 
to the effect of nanowire shrinkage. However in nanowire case [19] this shrinkage causes moving toward the core region of $\mathrm{Zn}$ canion site but not in the case of NP since there is stronger effect of relaxation occurs in axial directions due to the polar termination surface. So the NP will have a tendency to become bi-concave faceted in the perpendicular to the prisms axe direction and convex along it, see Fig. 3. Note the Zn-facet is much more deformed in comparison to the O-facet.

Figure 4 shows the shortening the distance along prismatic axe direction in comparison with bulk material and nanowire case. Note that in nanowire case there only relaxation in outer free surface so that this causes the wire be elongated along the prisms axe direction. Figures 2 and 4 also shows that surface relaxation occurs on a typical length of about $1 \mathrm{~nm}$ in the vicinity of surfaces region.
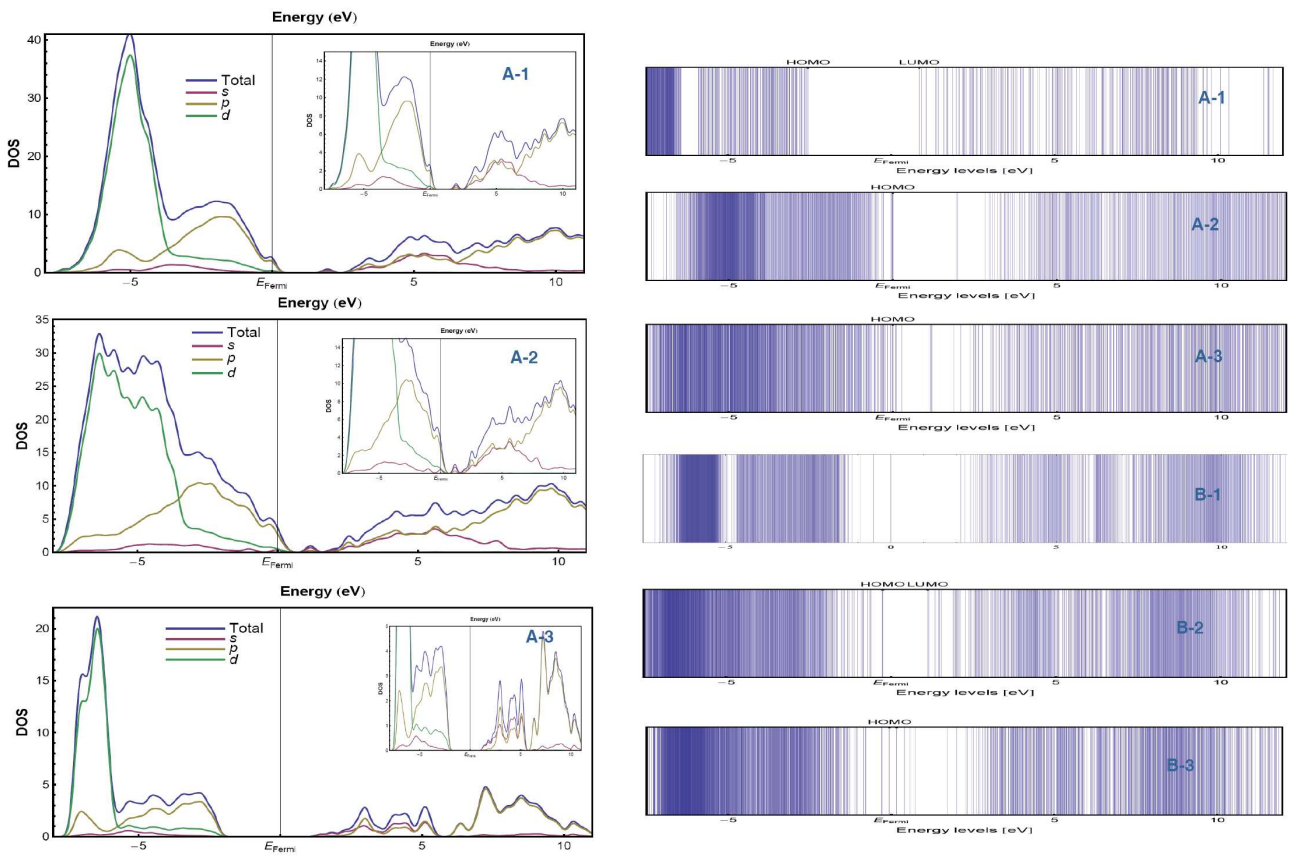

Fig. 5. Left column: Density of State (DOS) of Series A NPs. Right column: Spectral lines of NPs.

\section{III.2. Spectral structure and Density of States}

Fig. 5 shows the DOS analysis representatively for the sample series A (left column). These DOS build from the set of energy levels of spectral lines of the NPs series showing in the right column with explicit position of the Highest Occupied Molecular State (HOMO) and the Lowest Unoccupied Molecular State (LUMO). Fig. 6 shows a clear tendency to shorten the gap i.e. between HOMO and LUMO from the closed to infinite nanowire value to 0 showing the change from semiconductor (A-1) to the semi metallic (A-2) and metallic (A-3) behavior while increasing the NPs prismatic length (along with the growing of the facet relaxation deform). 

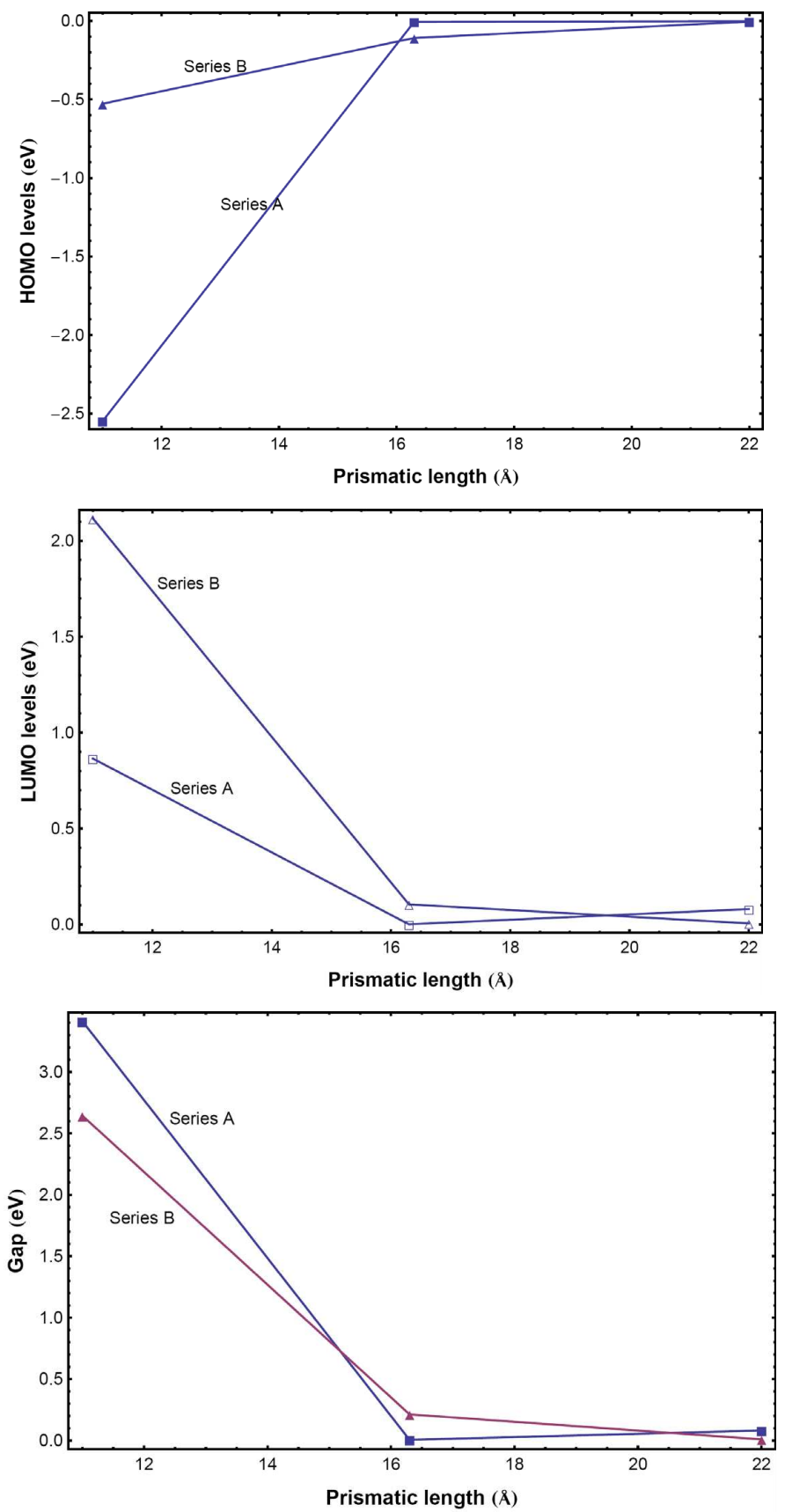

Fig. 6. HOMO, LUMO levels (Fermi level is set to zero) and energy gap of studied NPs series. 


\section{CONCLUSIONS}

We have studied the NPs hexagonal facet with different size (diameter and length). Here we observed the two main stabilization mechanisms of polar $\mathrm{ZnO}$ surfaces firstly have been proposed [2] as: (1) reconstruction of the surface and (2) metallization of the surface by charge transfer. The size dependence electronic properties are explained by the DOS

and the spectral analysis showing the role of the surface dipole and surface reconstruction. This assumption agrees with the experimental result[3].

\section{ACKNOWLEDGMENT}

This work was supported by the Vietnamese NAFOSTED program No. 103.02.100.09.

\section{REFERENCES}

[1] R F Khairutdinov, Russian Chemical Reviews 67 (2) (1998) 109-122.

[2] N. Combe, P. Chassaing, and F. Demangeot, Phys. Rev. B 79 (2009) 045408.

[3] P.-M. Chassaing, F. Demangeot, N. Combe, L. Saint-Macary, M. L. Kahn, and B. Chaudret, Phys. Rev. B 79 (2009) 155314.

[4] J. Park, H. Zheng, Y. Jun, and A. P. Alivisatos, J. Am. Chem. Soc. 131 (2009) 13943.

[5] B. Wang, J. Zhao, J. Jia, D. Shi, J. Wan, G. Wang, H. Xu, Appl. Phys. Lett., 93 (2008) 021918.

[6] M. Elstner, D. Porezag, G. Jungnickel, J. Elsner, M. Haugk, Th. Frauenheim, S. Suhai, G. Deifert, Phys. Rev. B, 58 (1998) 7260.

[7] C. Kohler, G. Seifert, Th. Frauenheim, Chem. Phys., 309 (2005) 23.

[8] M. Elstner, D. Porezag, G. Jungnickel, J. Elsner, M. Haugk, T.; B. Aradi, B. Hourahine and Th. Frauenheim, J. Phys. Chem. A, 111 (2007) 5678.

[9] B. Aradi, B. Hourahine, Th. Frauenheim, J. Phys. Chem. A, 111 (2007) 5678-5684.

[10] Ney H. Moreira, Grygoriy Dolgonos, Balint Aradi, Andreia L. da Rosa, Thomas Frauenheim, J. Chem. Theory Comput., 5 (2009) 605-614.

[11] R.Q. Zhang, X. Zhang, A.L. Rosa, Th. Frauenheim, Nanotechnology, 18 (2007) 485713.

[12] W. Fan, H. Xu, A. L. Rosa, Th. Frauenheim, R. Q. Zhang, Phys. Rev. B, 76 (2007) 73302.

[13] I.L.Damien J. Carter, Julian D Gale, Bernard Delley, Catherine Stampfl, Phys. Rev. B, 77 (2008) 15349 .

[14] Guofeng Wang, Xiaodong Li, Appl. Phys. Lett, 91 (2007) 231912.

[15] 15. C. Q. Chen, Y. Shi, Y. S. Zhang, J. Zhu, and Y. J. Yan, Phys. Rev. Lett., 96 (2006) 075505.

[16] H. Xu, A. L. Rosa, Th. Frauenheim, R. Q. Zhang, S. T. Lee, Applied Phys. Lett., 91 (2007) 31914.

[17] Y. Maoa, J. Zhonga, Y. Chen, Physica E, 40 (2008) 499-502.

[18] Y.R. Yang, X.H. Yan, Y. Xiao, Z.H. Guo, Chem. Phys. Lett., 446 (2007) 98-102.

[19] V.N. Tuoc,, Comp. Mat. Sci., 49 (2010) 161-169.

Received 15 September 2010. 